Diagnostic was suspected on hypotonia with poor suck in the neonatal period in the first case, hypotonia with history of feeding difficulty and psychomotor developmental delay in the second case and hyperphagia with obesity in the third case.

Physical exam showed facial dysmorphy in 1 case, bilateral cryptorchidism in the 3 cases and obesity $(\mathrm{BMI}=34.3)$ in the third case.

Chromosome analysis with fluorescence in situ hybridization (FISH) confirmed the diagnosis with identification of the deletion $15 \mathrm{q} 11.2-\mathrm{q} 13$ in the three cases.

The average retreat was 2 years; the evolution was marked by morbid obesity $(\mathrm{BMI}=57)$ with hypertension and psychiatric disturbance with hyperactivity in the third case and significant weight gain at the age of 10 months in the second case.

Conclusion Prader Willi must be suspected in all newborns with unexplained persistent hypotonia and confirmed by chromosome analysis. Early diagnosis is important to effective long-term management.

\section{PO-0367 FORMULA INTOLERANCE AND ORTHODOX DIETARY LAWS - A PASSOVER CONNEXION}

K Kaufman. Psychiatry and Neurology, Rutgers Robert Wood Johnson Medical School, New Brunswick, USA

\subsection{6/archdischild-2014-307384.1015}

Background When neonates and infants are not breastfed or require supplemental feedings, American Academy of Paediatrics recommends iron-supplemented formula. Cow's milk-based formula can be allergenic; however, this allergic response may be secondary to formula components as opposed to milk. The allergen may be inscrutable leading to false assumptions. In this case, an unusual religious clue solved such mystery.

Method Case analysis

Results An orthodox Jewish infant initially breastfed was transitioned to formula. Enfamil 4ounces q4hours caused upper respiratory mucus build-up directly post 'bottle-time'; formula was changed to ProSobee (soy-based) with resolution of 'formula intolerance.' At 12 months, cow's milk was initiated without allergic response. At 15 months, when diet included solid foods, she developed a peri-oral rash temporally associated with meals in her parents' home and following a meal in another family's home. Initial assessment revealed no new foods during these meals; however, this new allergy occurred during Passover, with strict dietary laws, suggesting a common new food. Ashkenazi Passover dietary laws preclude kitniyot (legumes, derivatives and associated crops including corn/soybeans/corn oil/soybean oil). Common Passover cooking oils include safflower oil. As both families were Ashkenazi Jews, the apparent common new food was safflower oil used in both homes. Safflower oil is an Enfamil ingredient. The presumptive allergen common to formula and Passover meals was safflower oil.

Conclusions Allergen determination may be secondary to fortuitous double exposure. Complete clinical histories need to allow for possible double exposure with willingness to consider cultural, religious, dietary law, and holiday dietary law factors.

\section{PO-0368 WITHDRAWN}

\section{PO-0369 A NIEMANN-PICK DISEASE TYPE C (NP-C) SUSPICION INDEX TOOL TO AID DIAGNOSIS IN PAEDIATRIC PATIENTS}

${ }^{1} \mathrm{CM}$ Lourenco, ${ }^{2} \mathrm{M}$ Pineda, ${ }^{3} \mathrm{E}$ Mengel, ${ }^{4} \mathrm{~B}$ Heron, ${ }^{5} \mathrm{I}$ Imrie, ${ }^{6} \mathrm{SA}$ Jones, ${ }^{7} \mathrm{~V}$ van der Linden, ${ }^{8 \mathrm{H}}$ Jahnova, ${ }^{9} \mathrm{P}$ Jesina, ${ }^{10} \mathrm{P}$ Karimzadeh, ${ }^{11} \mathrm{~V}$ Valayannopoulos, ${ }^{12} \mathrm{JM}$ Torres, ${ }^{13} \mathrm{~S}$ Kolb. ${ }^{1}$ Clinical Genetics, University of Sao Paulo, São Paulo, Brazil; ${ }^{2}$ Fundación Hospital Sant Joan de Deu CIBERER, Instituto de Salud Carlos III, Barcelona, Spain; ${ }^{3} V i l l a$ Metabolica, ZKJM MC University Mainz, Mainz, Germany; ${ }^{4}$ Centre Référence Des Maladies Lysosomales, CHU Trousseau APHP, Paris, France; ${ }^{5}$ Central Manchester and Manchester Children's Foundation Trust, University of Manchester, Manchester, UK; ${ }^{6}$ Manchester Centre for Genomic Medicine, Central Manchester Universty Hospitals Foundation Trust, Manchester, UK; ${ }^{7}$ Paediatrician, Association for Assistance to Disabled Children (AACD), Pernambuco, Brazil; ${ }^{8}$ Institute of Inherited Metabolic Disorders, Charles University, Praque, Czech Republic; ${ }^{9}$ Department of Pediatrics and Adolescent Medicine, Charles University, Prague, Czech Republic; ${ }^{10}$ Pediatric Neurology Research Center, Shahid Beheshti University of Medical Sciences, Tehran, Iran; ${ }^{11}$ Centre Référence Des Maladies Héréditaires Du Métabolisme de l'Enfant Et de l'Adulte, Hôpital Universitaire NeckerEnfants Malades, Paris, France; ${ }^{12}$ Biostatistics, Syntax for Science SL, Basel, Switzerland; ${ }^{13}$ Global Medical Lead, Actelion Pharmaceuticals Ltd, Allschwil, Switzerland

\subsection{6/archdischild-2014-307384.1016}

NP-C is a lysosomal lipid storage disorder caused by mutations in NPC1 or NPC2 genes. NP-C can present with a range of visceral, neurological and psychiatric symptoms that vary with age. A suspicion index (SI) tool was developed to assist clinicians achieve early diagnosis. The tool accurately predicts NP-C in patients $>4$ years of age but performs poorly in paediatric $(\leq 4$ years) patients. The present study aimed to utilise the characteristic symptomatology of NP-C in paediatric patients to develop a novel tool to assist paediatricians to identify patients for NP-C testing.

Paediatric patients were classified according to diagnosis: NP-C suspected and confirmed $(n=106)$; NP-C suspected but negative ( $\mathrm{n}=31$ ); control (no suspicion of NP-C; $\mathrm{n}=63$ ). Symptomatology data were collected retrospectively by questionnaire and summarised descriptively. The relationships between individual symptoms and likelihood of confirmed diagnosis of NP-C were defined by statistical modelling. The final tool was developed iteratively using combinations of symptoms until optimal discriminatory power was achieved.

The characteristic symptomatology of paediatric NP-C patients was identified; visceral symptoms were more prominent compared with older patients. The new tool discriminates well between NP-C confirmed, NP-C negative and control subjects. Statistical analysis demonstrates superior sensitivity and specificity of the paediatric tool compared to the original tool. The newly developed paediatric NP-C SI tool will help paediatricians to identify more paediatric patients with a high suspicion of NP-C, leading to more referrals for specialist testing thus improving early diagnosis and management of NPC-disease in paediatric patients.

Supported by Actelion Pharmaceuticals Ltd.

\section{PO-0370 GERM CELLS INDUCED FROM HUMAN UMBILICAL CORD MESENCHYMAL CELL-DERIVED INDUCED PLURIPOTENT STEM CELLS BY BMP4}

${ }^{1} \mathrm{~L} \mathrm{Ma},{ }^{2} \mathrm{~T}$ Wang, ${ }^{3} \mathrm{YB}$ Chen. ${ }^{1}$ Pediatrics, The Second Affiliated Hospital of Shantou University Medical College, Shantou, China; ${ }^{2}$ Pediatrics, Capital Institue of Pediatrics, Beijing, China; ${ }^{3}$ Pediatrics, Guangdong Maternity and Child Health Care Hospital, Guangzhou, China

10.1136/archdischild-2014-307384.1017 
Objective To reprogramme the induced pluripotent stem (iPS) cells from Human umbilical cord mesenchymal cells (HuMSCs) and induce the iPS cells into germ cells by BMP4.

Methods OCT4, SOX2, Klf4,c-myc, Nanog, Lin28 were transfected into HuMSCs with lentivirus to reprogram HuMSCs into iPS cells. Morphological observation, Alkaline Phosphatase staining, karyotype analysis, RT-PCR, immunofluorescence staining, tumour formation in vivo and embryniod body formation in vitro were performed to examine the pluripotency of the iPS cell lines. Then we induced one of the iPS cells lines into germ cells by BMP4. Gene expression was measured by qRT-PCR at days $0,3,7,10$ and 14. Early-stage germ specific protein VASA and meiosis specific protein SYCP3 were assesssed by immunofluorescence staining.

Result We obtained two iPS cell lines completely reprogrammed, HuMSC-iPS1 and HuMSC-iPS2. HuMSC-iPS1 expresses germ cell markers at undifferentiated state. BMP4 can upregulate germ cell markers at different time points highly while the spontaneous differentiation just upregulate DPPA3, DAZL and VASA modestly at day 3. However, all of these genes were downregulated at day 14. VASA and SYCP3 immunofluorescence staining indicates there is a high VASA expression in BMP4 induced group in contrast to low expression in the spontaneous group at day 7. Meanwhile, there is a modest SYCP3 fluorescence in BMP4 induced group in contrast to no immunofluoresence in the spontaneous group.

Conclusion This system can reprogram HuMSCs into iPS cells effectively. The MSC- iPS1 can differentiate into early germ cells spontaneously while the germ cells induced by BMP4 can enter meiosis.

\section{PO-0371 CLINICAL FEATURES AND CLINICAL OUTCOME IN CHILDREN WITH CHRONIC RECURRENT MULTIFOCAL OSTEOMYELITIS: THE EXPERIENCE OF A TERTIARY PAEDIATRIC CENTRE}

${ }^{1} \mathrm{~L}$ Martins, ${ }^{1} \mathrm{C}$ Alves, ${ }^{1} \mathrm{~B}$ Balacó, ${ }^{2} \mathrm{P}$ Estanqueiro, ${ }^{2} \mathrm{MB}$ Salgado, ${ }^{1} \mathrm{G}$ Matos. 'Department of Pediatric Orthopedics, Centro Hospitalar Universitário de Coimbra EPE-Hospital Pediátrico, Coimbra, Portugal; ${ }^{2}$ Department of Pediatrics Pediatric Rheumatology Unit, Centro Hospitalar Universitário de Coimbra EPE- Hospital Pediátrico, Coimbra, Portugal

10.1136/archdischild-2014-307384.1018

Introduction Chronic recurrent multifocal osteomyelitis (CRMO) is a rare auto-inflamatory bone disease, primarily affecting children and adolescents.

Objective To determine the clinical features and functional outcomes of children with CRMO treated between 1995 and 2013 in a tertiary paediatric hospital.

Methods We retrospectively reviewed clinical features of children with CRMO. Outcomes were obtained through telephonic interview. The Health Assessment Questionnaire (HAQ) disability índex and the Quick Dash questionnaire were applied. Upper limb disability of patients with clavicular involvement was compared with a control group of children who had a previous forearm fracture.

Results Nine patients were assessed (6 males and 3 females). Mean age at onset was 8 years; mean age at diagnosis 10,3 years. Mean number of bony lesions were 3 at onset and 3,4 over the disease course. The more frequently affected site was clavicle $(55,6 \%)$. Pain was the most frequent clinical sign of the disease $(88,9 \%)$ associated with swelling $(77,8 \%)$. Patients were treated with steroids $(88,9 \%)$, methotrexate $(44,4 \%)$, pamidronate $(22,2 \%)$ and NSAID (100\%). Five patients presented residual bone deformity (including two with inactive disease). All patients reported no physical disability as judged by the HAQ 01. However, patients with clavicular involvement (5) scored significantly worst on Quick Dash when compared to the control group ( $\mathrm{p}=0,015)$.

Conclusions Although the clinical outcome of children with CRMO is generally considered good, using an upper-extremity outcome measure (Quick DASH) allows us to understand that these patients experience upper limb difficulties in their daily lives.

\section{PO-0372 AUTOIMMUNE HEPATITIS: BIOCHEMICAL AND CLINICAL REVIEW OF A SERIES OF 4 CASES}

M Muñoz-Garcia, AM Arevalo-Garrido, MA Perez-Parras, J de la Cruz-Moreno. Department of Pediatrics, Complejo Hospitalario de Jaén, Jaén, Spain

\subsection{6/archdischild-2014-307384.1019}

The clinical spectrum of Autoinmune hepatitis (AH) is very wide, ranging from asymptomatic individuals with abnormal liver function to those with fulminant liver failure. The objective of this study was to analyse the clinical presentation, biochemical and histological diagnosis of a number of cases of $\mathrm{AH}$.

Methods between January-2006 and November-2013, we found 4 patients who were diagnosed $\mathrm{AH}$.

Results The median age was 11.9 years (2-13), predominantly female (75\%). The level of GOT and GPT were elevated, with a median of 259 IU (128-2451) and 309 IU (117-1449) respectively. The mean serum albumin was $3.9 \pm 0.3 \mathrm{~g} / \mathrm{dl}$ and the presence of $\operatorname{IgG}$ mean was $2391.5 \pm 628$. In all cases, the liver biopsy was performed to establish diagnosis. The anti-liver Kidney microsome type-1 (anti-LKM1) antibodies were present in all patients, and only in two of patients were found positive anti-smooth muscle (ASMA) antibodies. In 50\% were positive for antinuclear (ANA). Among the associated comorbidities found a case of hypothyroidism and vitiligo. Note that two of the four cases correspond to the same family, where molecular genetic study was carried out, with both sisters carrying HLA haplotypes DRB1 07. DRB1 14/DQB1 02. DQB105. In the majority of patients had non-specific symptoms, and only one patient presented jaundiced with abdominal pain. At follow-up, all patients are alive and there were not progression to cirrhosis or fulminant liver failure

Conclusion In our series, the autoimmune hepatitis was characterised predominant in female, with non-specific symptoms, had levels of transaminases elevated and associated with other autoimmune pathologies.

\section{PO-0373 LEAD, MERCURY, CADMIUM LEVELS IN BREAST MILK AND INFANT HAIR IN THE LATE PERIOD OF LACTATION FROM ANKARA, TURKEY}

${ }^{1}$ E Orun, ${ }^{2} S$ Yalcin. ${ }^{1}$ Pediatrics, Turgut Ozal University School of Medicine, Ankara, Turkey; ${ }^{2}$ Social Pediatrics, Hacettepe University Faculty of Medicine, Ankara, Turkey

\subsection{6/archdischild-2014-307384.1020}

Aim Lead $(\mathrm{Pb})$, mercury $(\mathrm{Hg})$, cadmium $(\mathrm{Cd})$ are found widely in environment and stay in nature for a long time. These toxic chemicals have some hazardous effects on child health. The purpose of this study are to determine the levels of heavy metals $(\mathrm{Pb}, \mathrm{Hg}, \mathrm{Cd})$ in breast milk and infant hair, related 